\title{
Sharp error bounds in approximating the Riemann-Stieltjes integral by a generalised trapezoid formula and applications
}

Pietro Cerone ${ }^{1 *}$ and Sever S Dragomir2,3

\section{"Correspondence:}

p.cerone@latrobe.edu.au

${ }^{1}$ Department of Mathematics and

Statistics, La Trobe University,

Melbourne, VIC 3086, Australia

Full list of author information is

available at the end of the article

\begin{abstract}
Sharp error bounds in approximating the Riemann-Stieltjes integral $\int_{a}^{b} f(t) d u(t)$ with the generalised trapezoid formula $f(b)\left[u(b)-\frac{1}{b-a} \int_{a}^{b} u(s) d s\right]+f(a)\left[\frac{1}{b-a} \int_{a}^{b} u(s) d s-u(a)\right]$ are given for various pairs $(f, u)$ of functions. Applications for weighted integrals are also provided.

MSC: 26D15; 26D10;41A55

Keywords: Riemann-Stieltjes integral; trapezoid rule; integral inequalities; weighted integrals
\end{abstract}

\section{Introduction}

In [1], in order to approximate the Riemann-Stieltjes integral $\int_{a}^{b} f(t) d u(t)$ by the generalised trapezoid formula

$$
[u(b)-u(x)] f(b)+[u(x)-u(a)] f(a), \quad x \in[a, b],
$$

the authors considered the error functional

$$
T(f, u ; a, b ; x):=\int_{a}^{b} f(t) d u(t)-[u(b)-u(x)] f(b)-[u(x)-u(a)] f(a)
$$

and proved that

$$
|T(f, u ; a, b ; x)| \leq H\left[\frac{1}{2}(b-a)+\left|x-\frac{a+b}{2}\right|\right]^{r} \bigvee_{a}^{b}(f), \quad x \in[a, b],
$$

provided that $f:[a, b] \rightarrow \mathbb{R}$ is of bounded variation on $[a, b]$ and $u$ is of $r-H$-Hölder type, that is, $u:[a, b] \rightarrow \mathbb{R}$ satisfies the condition $|u(t)-u(s)| \leq H|t-s|^{r}$ for any $t, s \in[a, b]$, where $r \in(0,1]$ and $H>0$ are given.

The dual case, namely, when $f$ is of $q-K$-Hölder type and $u$ is of bounded variation, has been considered by the authors in [2] in which they obtained the bound:

$$
\begin{aligned}
& |T(f, u ; a, b ; x)| \\
& \quad \leq K\left[(x-a)^{q} \bigvee_{a}^{x}(u)+(b-x)^{q} \bigvee_{x}^{b}(u)\right]
\end{aligned}
$$

(0) 2013 Cerone and Dragomir; licensee Springer. This is an Open Access article distributed under the terms of the Creative Commons Attribution License (http://creativecommons.org/licenses/by/2.0), which permits unrestricted use, distribution, and reproduction in any medium, provided the original work is properly cited. 


$$
\leq\left\{\begin{array}{l}
K\left[(x-a)^{q}+(b-x)^{q}\right]\left[\frac{1}{2} \bigvee_{a}^{b}(u)+\frac{1}{2}\left|\bigvee_{a}^{x}(u)-\bigvee_{x}^{b}(u)\right|\right] \\
K\left[(x-a)^{q \alpha}+(b-x)^{q \alpha}\right]^{\frac{1}{\alpha}}\left[\left[\bigvee_{a}^{x}(u)\right]^{\beta}-\left[\bigvee_{x}^{b}(u)\right]^{\beta}\right]^{\frac{1}{\beta}} \\
\quad \text { if } \alpha>1, \frac{1}{\alpha}+\frac{1}{\beta}=1 \\
K\left[\frac{1}{2}(b-a)+\left|x-\frac{a+b}{2}\right|\right]^{q} \bigvee_{a}^{b}(u)
\end{array}\right.
$$

for any $x \in[a, b]$.

The case where $f$ is monotonic and $u$ is of $r$-H-Hölder type, which provides a refinement for (1.3), and respectively the case where $u$ is monotonic and $f$ of $q$ - $K$-Hölder type were considered by Cheung and Dragomir in [3], while the case where one function was of Hölder type and the other was Lipschitzian was considered in [4]. For other recent results in estimating the error $T(f, u ; a, b, x)$ for absolutely continuous integrands $f$ and integrators $u$ of bounded variation, see [5] and [6].

The main aim of the present paper is to investigate the error bounds in approximating the Stieltjes integral by a different generalised trapezoid rule than the one from (1.1) in which the value $u(x), x \in[a, b]$ is replaced with the integral mean $\frac{1}{b-a} \int_{a}^{b} u(s) d s$. Applications in approximating the weighted integrals $\int_{a}^{b} h(t) f(t) d t$ are also provided.

\section{Representation results}

We consider the following error functional $T_{g}(f ; u)$ in approximating the Riemann-Stieltjes integral $\int_{a}^{b} f(t) d u(t)$ by the generalised trapezoid formula:

$$
\begin{aligned}
f(b)[u(b) & \left.-\frac{1}{b-a} \int_{a}^{b} u(t) d t\right]+f(a)\left[\frac{1}{b-a} \int_{a}^{b} u(t) d t-u(a)\right] \\
T_{g}(f ; u):= & f(b)\left[u(b)-\frac{1}{b-a} \int_{a}^{b} u(t) d t\right] \\
& +f(a)\left[\frac{1}{b-a} \int_{a}^{b} u(t) d t-u(a)\right]-\int_{a}^{b} f(t) d u(t) .
\end{aligned}
$$

If we consider the associated functions $\Phi_{f}, \Gamma_{f}$ and $\Delta_{f}$ defined by

$$
\begin{aligned}
& \Phi_{f}(t):=\frac{(t-a) f(b)+(b-t) f(a)}{b-a}-f(t), \quad t \in[a, b] \\
& \Gamma_{f}(t):=(t-a)[f(b)-f(t)]-(b-t)[f(t)-f(a)], \quad t \in[a, b]
\end{aligned}
$$

and

$$
\Delta_{f}(t):=\frac{f(b)-f(t)}{b-t}-\frac{f(t)-f(a)}{t-a}, \quad t \in(a, b),
$$

then we observe that

$$
\Phi_{f}(t)=\frac{1}{b-a} \Gamma_{f}(t)=\frac{(b-t)(t-a)}{b-a} \Delta_{f}(t) \quad \text { for any } t \in(a, b)
$$

The following representation result can be stated. 
Theorem 1 Let $f, u:[a, b] \rightarrow \mathbb{R}$ be bounded on $[a, b]$ and such that the Riemann-Stieltjes integral $\int_{a}^{b} f(t) d u(t)$ and the Riemann integral $\int_{a}^{b} u(t) d t$ exist. Then we have the identities

$$
\begin{aligned}
T_{g}(f ; u) & =\int_{a}^{b} \Phi_{f}(t) d u(t) \\
& =\frac{1}{b-a} \int_{a}^{b} \Gamma_{f}(t) d u(t) \\
& =\frac{1}{b-a} \int_{a}^{b}(b-t)(t-a) \Delta_{f}(t) d u(t) \\
& =D(u ; f),
\end{aligned}
$$

where

$$
D(u ; f)=\int_{a}^{b} u(t) d f(t)-[f(b)-f(a)] \cdot \frac{1}{b-a} \int_{a}^{b} u(t) d t .
$$

Proof Integrating the Riemann-Stieltjes integral by parts, we have

$$
\begin{aligned}
\int_{a}^{b} & \Phi_{f}(t) d u(t) \\
= & \int_{a}^{b}\left[\frac{f(a)(b-t)+f(b)(t-a)}{b-a}-f(t)\right] d u(t) \\
= & \frac{1}{b-a}\left\{\left.[f(a)(b-t)+f(b)(t-a)] u(t)\right|_{a} ^{b}\right. \\
& \left.-\int_{a}^{b} u(t) d[f(a)(b-t)+f(b)(t-a)]\right\}-\int_{a}^{b} f(t) d u(t) \\
= & \frac{1}{b-a}\left\{[f(b) u(b)-f(a) u(a)](b-a)-[f(b)-f(a)] \int_{a}^{b} u(t) d t\right\}-\int_{a}^{b} f(t) d u(t) \\
= & f(b)\left[u(b)-\frac{1}{b-a} \int_{a}^{b} u(t) d t\right] \\
& +f(a)\left[\frac{1}{b-a} \int_{a}^{b} u(t) d t-u(a)\right]-\int_{a}^{b} f(t) d u(t) \\
= & T_{g}(f ; u),
\end{aligned}
$$

and the first equality in (2.3) is proved.

The second and third identity is obvious by the relation (2.2).

For the last equality, we use the fact that for any $g, h:[a, b] \rightarrow \mathbb{R}$ bounded functions for which the Riemann-Stieltjes integral $\int_{a}^{b} h(t) d g(t)$ and the Riemann integral $\int_{a}^{b} g(t) d t$ exist, we have the representation (see, for instance, [7])

$$
D(g ; h)=\int_{a}^{b} \Phi_{h}(t) d g(t)
$$

The proof is now complete.

In the case where $u$ is an integral, the following identity can be stated. 
Corollary 1 Let $p, h:[a, b] \rightarrow \mathbb{R}$ be continuous on $[a, b]$ and $f:[a, b] \rightarrow \mathbb{R}$ be Riemann integrable. Then we have the identity

$$
\begin{aligned}
T_{g}\left(f ; \int_{a} p h\right)= & \frac{1}{b-a}\left[f(b) \cdot \int_{a}^{b}(t-a) p(t) h(t) d t+f(a) \cdot \int_{a}^{b}(b-t) p(t) h(t) d t\right] \\
& -\int_{a}^{b} p(t) f(t) h(t) d t \\
= & \int_{a}^{b} \Phi_{f}(t) p(t) h(t) d t .
\end{aligned}
$$

Proof Since $p$ and $h$ are continuous, the function $u(t)=\int_{a}^{t} p(s) h(s) d s$ is differentiable and $u^{\prime}(t)=p(t) h(t)$ for each $t \in(a, b)$.

Integrating by parts, we have

$$
\begin{aligned}
\int_{a}^{b} u(t) d t & =\left.\left(\int_{a}^{t} p(s) h(s) d s\right) \cdot t\right|_{a} ^{b}-\int_{a}^{b} t p(t) h(t) d t \\
& =b \int_{a}^{b} p(s) h(s) d s-\int_{a}^{b} t p(t) h(t) d t \\
& =\int_{a}^{b}(b-t) p(t) h(t) d t .
\end{aligned}
$$

Since

$$
\begin{aligned}
u(b)-\frac{1}{b-a} \int_{a}^{b} u(t) d t & =\int_{a}^{b} p(t) h(t) d t-\frac{1}{b-a} \int_{a}^{b}(b-t) p(t) h(t) d t \\
& =\frac{1}{b-a} \int_{a}^{b}(t-a) p(t) h(t) d t
\end{aligned}
$$

then, by the definition of $T_{g}$ in (2.1), we deduce the first part of (2.6).

The second part of (2.6) follows by (2.3).

Remark 1 In the particular case $p(t)=1, t \in[a, b]$, we have the equality

$$
\begin{aligned}
T_{g}\left(f ; \int_{a} h\right) & =\frac{1}{b-a}\left[f(b) \cdot \int_{a}^{b}(t-a) h(t) d t+f(a) \cdot \int_{a}^{b}(b-t) h(t) d t\right]-\int_{a}^{b} f(t) h(t) d t \\
& =\int_{a}^{b} \Phi_{f}(t) h(t) d t=\frac{1}{b-a} \int_{a}^{b} \Gamma_{f}(t) h(t) d t
\end{aligned}
$$

\section{Some inequalities for $f$-convex}

The following result concerning the nonnegativity of the error functional $T_{g}(\cdot ; \cdot)$ can be stated.

Theorem 2 If $u$ is monotonic nonincreasing and $f:[a, b] \rightarrow \mathbb{R}$ is such that the RiemannStieltjes integral $\int_{a}^{b} f(t) d u(t)$ exists and

$$
\frac{f(b)-f(t)}{b-t} \geq \frac{f(t)-f(a)}{t-a} \text { for any } t \in(a, b),
$$


then $T_{g}(f ; u) \geq 0$ or, equivalently,

$$
f(b)\left[u(b)-\frac{1}{b-a} \int_{a}^{b} u(t) d t\right]+f(a)\left[\frac{1}{b-a} \int_{a}^{b} u(t) d t-u(a)\right] \geq \int_{a}^{b} f(t) d u(t) .
$$

A sufficient condition for (3.1) to hold is that $f$ is convex on $[a, b]$.

Proof The condition (3.1) is equivalent with the fact that $\Delta_{f}(t) \geq 0$ for any $t \in(a, b)$ and then, by the equality

$$
T_{g}(f ; u)=\frac{1}{b-a} \int_{a}^{b}(b-t)(t-a) \Delta_{f}(t) d u(t),
$$

we deduce that $T_{g}(f ; u) \geq 0$.

If $f$ is convex, then

$$
\frac{t-a}{b-a} f(b)+\frac{b-t}{b-a} f(a) \geq f\left[\left(\frac{t-a}{b-a}\right) b+\left(\frac{b-t}{b-a}\right) a\right]=f(t),
$$

which shows that $\Phi_{f}(t) \geq 0$, namely, the condition (3.1) is satisfied.

Corollary 2 Let $p, h:[a, b] \rightarrow \mathbb{R}$ be continuous on $[a, b]$ and $f:[a, b] \rightarrow \mathbb{R}$ be Riemann integrable. If $p(t) h(t) \geq 0$ for any $t \in[a, b]$ and $f$ satisfies (3.1) or, sufficiently, $f$ is convex on $[a, b]$, then

$$
\begin{aligned}
& \frac{1}{b-a}\left[f(b) \cdot \int_{a}^{b}(t-a) p(t) h(t) d t+f(a) \cdot \int_{a}^{b}(b-t) p(t) h(t) d t\right] \\
& \geq \int_{a}^{b} p(t) f(t) h(t) d t .
\end{aligned}
$$

We are now able to provide some new results.

Theorem 3 Assume that p and h are continuous and synchronous (asynchronous) on $(a, b)$, i.e.,

$$
(p(t)-p(s))(h(t)-h(s)) \geq(\leq) 0 \text { for any } t, s \in[a, b] .
$$

Iff satisfies (3.1) and is Riemann integrable on $[a, b]$ (or sufficiently, $f$ is convex on $[a, b]$ ), then

$$
T_{g}\left(f ; \int_{a} p\right) \cdot T_{g}\left(f ; \int_{a} h\right) \leq(\geq) T_{g}\left(f ; \int_{a} 1\right) \cdot T_{g}\left(f ; \int_{a} p h\right),
$$

where

$$
T_{g}\left(f ; \int_{a} 1\right)=\frac{f(a)+f(b)}{2}(b-a)-\int_{a}^{b} f(t) d t .
$$

Proof We use the Čebyšev inequality

$$
\int_{a}^{b} \alpha(t) d t \int_{a}^{b} \alpha(t) p(t) h(t) d t \geq(\leq) \int_{a}^{b} \alpha(t) p(t) d t \int_{a}^{b} \alpha(t) h(t) d t,
$$


which holds for synchronous (asynchronous) functions $p, h$ and nonnegative $\alpha$ for which the involved integrals exist.

Now, on applying the Čebyšev inequality (3.7) for $\alpha(t)=\Phi_{f}(t) \geq 0$ and utilising the representation result (2.6), we deduce the desired inequality (3.5).

We also have the following theorem.

Theorem 4 Assume that $f:[a, b] \rightarrow \mathbb{R}$ is Riemann integrable and satisfies (3.1) (or sufficiently, $f$ is concave on $[a, b])$. Then, for $p, h:[a, b] \rightarrow \mathbb{R}$ continuous, we have

$$
\left|T_{g}\left(f ; \int_{a} p h\right)\right| \leq \sup _{t \in[a, b]}|h(t)| T_{g}\left(f ; \int_{a}|p|\right)
$$

and

$$
\left|T_{g}\left(f ; \int_{a} p h\right)\right| \leq\left[T_{g}\left(f ; \int_{a}|p|^{\alpha}\right)\right]^{\frac{1}{\alpha}}\left[T_{g}\left(f ; \int_{a}|h|^{\beta}\right)\right]^{\frac{1}{\beta}},
$$

where $\alpha>1, \frac{1}{\alpha}+\frac{1}{\beta}=1$. In particular, we have

$$
\left|T_{g}\left(f ; \int_{a} p h\right)\right|^{2} \leq T_{g}\left(f ; \int_{a}|p|^{2}\right) T_{g}\left(f ; \int_{a}|h|^{2}\right) .
$$

Proof Observe that

$$
\begin{aligned}
\left|T_{g}\left(f ; \int_{a} p h\right)\right| & =\left|\int_{a}^{b} \Phi_{f}(t) p(t) h(t) d t\right| \\
& \leq \int_{a}^{b}\left|\Phi_{f}(t) p(t) h(t)\right| d t \\
& =\int_{a}^{b} \Phi_{f}(t)|p(t)||h(t)| d t \\
& \leq \sup _{t \in[a, b]}|h(t)| \int_{a}^{b} \Phi_{f}(t)|p(t)| d t \\
& =\sup _{t \in[a, b]}|h(t)| T_{g}\left(f ; \int_{a}|p|\right)
\end{aligned}
$$

and the inequality (3.8) is proved.

Further, by the Hölder inequality, we also have

$$
\begin{aligned}
\left|T_{g}\left(f ; \int_{a} p h\right)\right| & \leq \int_{a}^{b} \Phi_{f}(t)|p(t)||h(t)| d t \\
& \leq\left(\int_{a}^{b} \Phi_{f}(t)|p(t)|^{\alpha} d t\right)^{\frac{1}{\alpha}}\left(\int_{a}^{b} \Phi_{f}(t)|h(t)|^{\beta} d t\right)^{\frac{1}{\beta}} \\
& =\left[T_{g}\left(f ; \int_{a}|p|^{\alpha}\right)\right]^{\frac{1}{\alpha}}\left[T_{g}\left(f ; \int_{a}|h|^{\beta}\right)\right]^{\frac{1}{\beta}}
\end{aligned}
$$

for $\alpha>1, \frac{1}{\alpha}+\frac{1}{\beta}=1$, and the theorem is proved. 
Remark 2 The above result can be useful for providing some error estimates in approximating the weighted integral $\int_{a}^{b} h(t) f(t) d t$ by the generalised trapezoid rule

$$
\frac{1}{b-a}\left[f(b) \cdot \int_{a}^{b}(t-a) h(t) d t+f(a) \cdot \int_{a}^{b}(b-t) h(t) d t\right]
$$

as follows:

$$
\begin{aligned}
& \left|\frac{1}{b-a}\left[f(b) \cdot \int_{a}^{b}(t-a) h(t) d t+f(a) \cdot \int_{a}^{b}(b-t) h(t) d t\right]-\int_{a}^{b} h(t) f(t) d t\right| \\
& \quad \leq \sup _{t \in[a, b]}|h(t)|\left[\frac{f(a)+f(b)}{2}(b-a)-\int_{a}^{b} f(t) d t\right],
\end{aligned}
$$

provided $f$ satisfies (3.1) and is Riemann integrable (or sufficiently, convex on $[a, b]$ ), which is continuous on $[a, b]$.

If $h(t)=|w(t)|^{\frac{1}{\beta}}, t \in[a, b]$, then for some $f$, we also have

$$
\begin{aligned}
\left.\left|\frac{1}{b-a}\left[f(b) \int_{a}^{b}(t-a)|w(t)|^{\frac{1}{\beta}} d t+f(a) \int_{a}^{b}(b-t)|w(t)|^{\frac{1}{\beta}} d t\right]-\int_{a}^{b}\right| w(t)\right|^{\frac{1}{\beta}} f(t) d t \mid \\
\leq\left[\frac{f(a)+f(b)}{2}(b-a)-\int_{a}^{b} f(t) d t\right]^{\frac{1}{\alpha}} \times\left\{\frac { 1 } { b - a } \left[f(b) \int_{a}^{b}(t-a)|w(t)| d t\right.\right. \\
\left.\left.\quad+f(a) \int_{a}^{b}(b-t)|w(t)| d t\right]-\int_{a}^{b}|w(t)| f(t) d t\right\}^{\frac{1}{\beta}},
\end{aligned}
$$

with $\alpha>1, \frac{1}{\alpha}+\frac{1}{\beta}=1$.

Finally, we can state the following Jensen type inequality for the error functional $T_{g}\left(f ; \int_{a}^{b} h\right)$.

Theorem 5 Assume $f:[a, b] \rightarrow \mathbb{R}$ is Riemann integrable and satisfies (3.1) (or sufficiently, $f$ is convex on $[a, b])$, while $h:[a, b] \rightarrow \mathbb{R}$ is continuous. If $F: \mathbb{R} \rightarrow \mathbb{R}$ is convex (concave), then

$$
F\left(\frac{T_{g}\left(f ; \int_{a}^{b} h\right)}{T_{g}\left(f ; \int_{a}^{b} 1\right)}\right) \leq(\geq) \frac{T_{g}\left(f ; \int_{a}^{b} F \circ h\right)}{T_{g}\left(f ; \int_{a}^{b} 1\right)} .
$$

Proof By the use of Jensen's integral inequality, we have

$$
F\left(\frac{\int_{a}^{b} \Phi_{f}(t) h(t) d t}{\int_{a}^{b} \Phi_{f}(t) d t}\right) \leq(\geq) \frac{\int_{a}^{b} \Phi_{f}(t) F(h(t)) d t}{\int_{a}^{b} \Phi_{f}(t) d t} .
$$

Since, by the identity (2.6), we have

$$
\int_{a}^{b} \Phi_{f}(t) F(h(t)) d t=T_{g}\left(f ; \int_{a}^{b} F \circ h\right)
$$

then (3.14) is equivalent with the desired result (3.13). 


\section{Sharp bounds via Grüss type inequalities}

Due to the identity (2.3), in which the error bound $T_{g}(f ; u)$ can be represented as $D(u ; f)$, where

$$
D(u ; f)=\int_{a}^{b} u(t) d f(t)-[f(b)-f(a)] \cdot \frac{1}{b-a} \int_{a}^{b} u(t) d t,
$$

is a Grüss type functional introduced in [8], any sharp bound for $D(u ; f)$ will be a sharp bound for $T_{g}(f ; u)$.

We can state the following result.

Theorem 6 Let $f, u:[a, b] \rightarrow \mathbb{R}$ be bounded functions on $[a, b]$.

(i) If there exist constants $n, N$ such that $n \leq u(t) \leq N$ for any $t \in[a, b]$, $u$ is Riemann integrable and $f$ is $K$-Lipschitzian $(K>0)$, then

$$
\left|T_{g}(f ; u)\right| \leq \frac{1}{2} K(N-n)(b-a) .
$$

The constant $\frac{1}{2}$ is best possible in (4.1).

(ii) If $f$ is of bounded variation and $u$ is $S$-Lipschitzian $(S>0)$, then

$$
\left|T_{g}(f ; u)\right| \leq \frac{1}{2} S(b-a) \bigvee_{a}^{b}(f)
$$

The constant $\frac{1}{2}$ is best possible in (4.2)

(iii) If $f$ is monotonic nondecreasing and $u$ is S-Lipschitzian, then

$$
\begin{aligned}
\left|T_{g}(f ; u)\right| & \leq \frac{1}{2} S(b-a)[f(b)-f(a)-P(f)] \\
& \leq \frac{1}{2} S(b-a)[f(b)-f(a)],
\end{aligned}
$$

where

$$
P(f)=\frac{4}{(b-a)^{2}} \int_{a}^{b}\left(t-\frac{a+b}{2}\right) f(t) d t .
$$

The constant $\frac{1}{2}$ is best possible in both inequalities.

(iv) If $f$ is monotonic nondecreasing and $u$ is of bounded variation and such that the Riemann-Stieltjes integral $\int_{a}^{b} f(t) d u(t)$ exists, then

$$
\left|T_{g}(f ; u)\right| \leq[f(b)-f(a)-Q(f)] \bigvee_{a}^{b}(u),
$$

where

$$
Q(f):=\frac{1}{b-a} \int_{a}^{b} \operatorname{sgn}\left(t-\frac{a+b}{2}\right) f(t) d t .
$$

The inequality (4.4) is sharp. 
(v) If $f$ is continuous and convex on $[a, b]$ and $u$ is of bounded variation on $[a, b]$, then

$$
\left|T_{g}(f ; u)\right| \leq \frac{1}{4}\left[f_{-}^{\prime}(b)-f_{+}^{\prime}(a)\right] \bigvee_{a}^{b}(u)
$$

The constant $\frac{1}{4}$ is sharp (iff $f_{-}^{\prime}(b)$ and $f_{+}^{\prime}(a)$ are finite).

(vi) If $f:[a, b] \rightarrow \mathbb{R}$ is continuous and convex on $[a, b]$ and $u$ is monotonic

nondecreasing on $[a, b]$, then

$$
\begin{aligned}
0 & \leq T_{g}(f ; u) \\
& \leq 2 \cdot \frac{f_{-}^{\prime}(b)-f_{+}^{\prime}(a)}{b-a} \cdot \int_{a}^{b}\left(t-\frac{a+b}{2}\right) u(t) d t \\
& \leq\left\{\begin{array}{l}
\frac{1}{2}\left[f_{-}^{\prime}(b)-f_{+}^{\prime}(a)\right] \max \{|u(a)|,|u(b)|\}(b-a) ; \\
\frac{1}{(q+1)^{1 / q}}\left[f_{-}^{\prime}(b)-f_{+}^{\prime}(a)\right]\|u\|_{p}(b-a)^{1 / q} \quad \text { if } p>1, \frac{1}{p}+\frac{1}{q}=1 ; \\
{\left[f_{-}^{\prime}(b)-f_{+}^{\prime}(a)\right]\|u\|_{1} .}
\end{array}\right.
\end{aligned}
$$

The constants 2 and $\frac{1}{2}$ are best possible in (4.6) (iff $f_{-}^{\prime}(b)$ and $f_{+}^{\prime}(a)$ are finite).

Proof The inequality (4.1) follows from the inequality (2.5) in [8] applied to $D(u ; f)$, while (4.2) comes from (1.3) of [9]. The inequalities (4.3) and (4.4) follow from [7], while (4.5) and (4.6) are valid via the inequalities (2.8) and (2.1) from [10] applied to the functional $D(u ; f)$. The details are omitted.

If we consider the error functional in approximating the weighted integral $\int_{a}^{b} h(t) f(t) d t$ by the generalised trapezoid formula,

$$
\frac{1}{b-a}\left[f(b) \cdot \int_{a}^{b}(t-a) h(t) d t+f(a) \cdot \int_{a}^{b}(b-t) h(t) d t\right],
$$

namely (see also (2.7)),

$$
\begin{aligned}
E(f ; h):= & T_{g}\left(f ; \int_{a}^{b} h\right) \\
= & \frac{1}{b-a}\left[f(b) \cdot \int_{a}^{b}(t-a) h(t) d t+f(a) \cdot \int_{a}^{b}(b-t) h(t) d t\right] \\
& -\int_{a}^{b} h(t) f(t) d t,
\end{aligned}
$$

then the following corollary provides various sharp bounds for the absolute value of $E(f ; h)$.

Corollary 3 Assume that $f$ and $u$ are Riemann integrable on $[a, b]$.

(i) If there exist constants $\gamma, \Gamma$ such that $\gamma \leq \int_{a}^{t} h(s) d s \leq \Gamma$ for each $t \in[a, b]$, and $f$ is $K$-Lipschitzian on $[a, b]$, then

$$
|E(f ; h)| \leq \frac{1}{2} K(\Gamma-\gamma)(b-a) .
$$

The constant $\frac{1}{2}$ is best possible in (4.8). 
(ii) If $f$ is of bounded variation and $|h(t)| \leq M$ for each $t \in[a, b]$, then

$$
|E(f ; h)| \leq \frac{1}{2} M(b-a) \bigvee_{a}^{b}(f)
$$

The constant $\frac{1}{2}$ is best possible in (4.9).

(iii) If $f$ is monotonic nondecreasing and $|h(t)| \leq M, t \in[a, b]$, then

$$
|E(f ; h)| \leq \frac{1}{2} M(b-a)[f(b)-f(a)-P(f)] \leq \frac{1}{2} M(b-a)[f(b)-f(a)]
$$

where $P(f)$ is defined in Theorem 6. The constant $\frac{1}{2}$ is sharp in both inequalities.

(iv) If $\mathrm{is}$ monotonic nondecreasing and $\int_{a}^{b}|h(t)| d t<\infty$, then

$$
|E(f ; h)| \leq[f(b)-f(a)-Q(f)] \int_{a}^{b}|h(t)| d t
$$

where $Q(f)$ is defined in Theorem 6. The inequality (4.11) is sharp.

(v) If $f$ is continuous and convex on $[a, b]$ and $\int_{a}^{b}|h(t)| d t<\infty$, then

$$
|E(f ; h)| \leq \frac{1}{4}\left[f_{-}^{\prime}(b)-f_{+}^{\prime}(a)\right] \int_{a}^{b}|h(t)| d t
$$

The constant $\frac{1}{4}$ is sharp (iff $f_{-}^{\prime}(b)$ and $f_{+}^{\prime}(a)$ are finite).

(vi) If $f:[a, b] \rightarrow \mathbb{R}$ is continuous and convex on $[a, b]$ and $h(t) \geq 0$ for $t \in[a, b]$, then

$$
\begin{aligned}
0 & \leq E(f ; h) \\
& \leq \frac{f_{-}^{\prime}(b)-f_{+}^{\prime}(a)}{b-a} \int_{a}^{b}(b-t)(t-a) h(t) d t \\
& \leq\left\{\begin{array}{l}
\frac{1}{2}\left[f_{-}^{\prime}(b)-f_{+}^{\prime}(a)\right](b-a) \int_{a}^{b} h(t) d t ; \\
\frac{1}{(q+1)^{1 / q}}\left[f_{-}^{\prime}(b)-f_{+}^{\prime}(a)\right]\left[\int_{a}^{b}\left(\int_{a}^{t} h(s) d s\right)^{p} d t\right]^{\frac{1}{p}}(b-a)^{1 / q} \\
i f p>1, \frac{1}{p}+\frac{1}{q}=1 ; \\
{\left[f_{-}^{\prime}(b)-f_{+}^{\prime}(a)\right] \int_{a}^{b}(b-t) h(t) d t .}
\end{array}\right.
\end{aligned}
$$

The first inequality in (4.13) is sharp (iff $f_{-}^{\prime}(b)$ and $f_{+}^{\prime}(a)$ are finite).

Proof We only prove the first inequality in (4.13).

Utilising the inequality (4.6) for $u(t)=\int_{a}^{t} h(s) d s$, we get

$$
0 \leq E(f ; h) \leq 2 \cdot \frac{f_{-}^{\prime}(b)-f_{+}^{\prime}(a)}{b-a} \int_{a}^{b}\left(t-\frac{a+b}{2}\right) \int_{a}^{t} h(s) d s d t
$$

However, on integrating by parts, we have

$$
\begin{aligned}
& \int_{a}^{b}\left(t-\frac{a+b}{2}\right) \int_{a}^{t} h(s) d s d t \\
& =\int_{a}^{b}\left(\int_{a}^{t} h(s) d s\right) d\left[\frac{1}{2}\left(t-\frac{a+b}{2}\right)^{2}\right]
\end{aligned}
$$




$$
\begin{aligned}
& =\left.\frac{1}{2}\left(t-\frac{a+b}{2}\right)^{2} \int_{a}^{t} h(s) d s\right|_{a} ^{b}-\frac{1}{2} \int_{a}^{b}\left(t-\frac{a+b}{2}\right)^{2} h(t) d t \\
& =\frac{1}{2}\left[\left(\frac{b-a}{2}\right)^{2} \int_{a}^{b} h(t) d t-\int_{a}^{b}\left(t-\frac{a+b}{2}\right)^{2} h(t) d t\right] \\
& =\frac{1}{2} \int_{a}^{b}\left[\left(\frac{b-a}{2}\right)^{2}-\left(t-\frac{a+b}{2}\right)^{2}\right] h(t) d t \\
& =\frac{1}{2} \int_{a}^{b}(b-t)(t-a) h(t) d t .
\end{aligned}
$$

The rest of the inequality is obvious.

\section{Competing interests}

The authors declare that they have no competing interests.

\section{Authors' contributions}

PC and SSD have contributed to all parts of the article. Both authors read and approved the final manuscript.

\section{Author details}

${ }^{1}$ Department of Mathematics and Statistics, La Trobe University, Melbourne, VIC 3086, Australia. ${ }^{2}$ Mathematics, College of Engineering \& Science, Victoria University, P.O. Box 14428, Melbourne, MC 8001, Australia. ${ }^{3}$ School of Computational and Applied Mathematics, University of the Witwatersrand, Private Bag 3, Johannesburg, Wits 2050, South Africa.

\section{Acknowledgements}

Most of the work for this article was undertaken while the first author was at Victoria University, Melbourne Australia.

Received: 18 July 2012 Accepted: 22 January 2013 Published: 18 February 2013

\section{References}

1. Dragomir, SS, Buse, C, Boldea, MV, Braescu, L: A generalization of the trapezoidal rule for the Riemann-Stieltjes integral and applications. Nonlinear Anal. Forum 6(2), 337-351 (2001)

2. Cerone, P, Dragomir, SS: New bounds for the three-point rule involving the Riemann-Stieltjes integral. In: Advances in Statistics, Combinatorics and Related Areas, pp. 53-62. World Scientific, River Edge (2002)

3. Cheung, W-S, Dragomir, SS: Two Ostrowski type inequalities for the Stieltjes integral of monotonic functions. Bull. Aust. Math. Soc. 75(2), 299-311 (2007)

4. Barnett, NS, Cheung, W-S, Dragomir, SS, Sofo, A: Ostrowski and trapezoid type inequalities for the Stieltjes integral with Lipschitzian integrands or integrators. RGMIA Res. Rep. Collect. 9, Article 9 (2006). http://rgmia.org/v9n4.php

5. Cerone, P, Dragomir, SS: Approximation of the Stieltjes integral and applications in numerical integration. Appl. Math. 51(1), 37-47 (2006)

6. Cerone, P, Cheung, WS, Dragomir, SS: On Ostrowski type inequalities for Stieltjes integrals with absolutely continuous integrands and integrators of bounded variation. Comput. Math. Appl. 54(2), 183-191 (2007)

7. Dragomir, SS: Inequalities of Grüss type for the Stieltjes integral and applications. Kragujev. J. Math. 26, 89-122 (2004)

8. Dragomir, SS, Fedotov, IA: An inequality of Grüss' type for Riemann-Stieltjes integral and applications for special means. Tamkang J. Math. 29(4), 287-292 (1998)

9. Dragomir, SS, Fedotov, I: A Grüss type inequality for mappings of bounded variation and applications to numerical analysis. Nonlinear Funct. Anal. Appl. 6(3), 425-438 (2001)

10. Dragomir, SS: Inequalities for Stieltjes integrals with convex integrators and applications. Appl. Math. Lett. 20(2), 123-130 (2007) 